\title{
Radially Defined Local Binary Patterns for Facial Expression Recognition
}

\author{
Megha V. Jonnalagedda \\ Associate Professor \\ Department of Information Technology \\ SGGS Institute of Engineering and Technology, \\ Vishnupuri, Nanded, M.S. India
}

\author{
Dharmpal D. Doye \\ Professor \\ Department of Electronics \& Telecomm ${ }^{n}$. Engineering \\ SGGS Institute of Engineering and Technology, \\ Vishnupuri, Nanded, M.S. India
}

\begin{abstract}
Automatic Facial Expression Recognition (FER) has attracted the attention of many researchers due to its potential applications. Extraction of proper and sufficient features from the facial image is the most important step for effective FER. As facial images can be differentiated from other textural images in the sense that they exhibit specific information as regards expressions around certain face regions (such as areas surrounding the eyes, nose and mouth), efforts need to be done on identifying the specific facial expression related information. Two different approaches have been envisaged and proposed in this paper taking into consideration the pixel value variations exhibited in different directions or regions when different expressions are subjected to feature extraction. The technique proposed basically finds Local Binary Pattern (LBP) like features but along the radial lines taken at specific angle. Another approach proposed considers the expression specific areas like eyes, nose and mouth for finding similar radial LBPs. The overall efficiency obtained is comparable to the popularly used LBP technique. Comparatively lesser time required for feature extraction and recognition as well as smaller region considered for feature extraction are promising aspects of the proposed techniques.
\end{abstract}

\section{General Terms}

Feature Extraction, Local Binary Patterns, Support Vector Machine

\section{Keywords}

Radial Local Binary Pattern (RLBP)

\section{INTRODUCTION}

A human face carries lot of information about the emotional and mental state of a person. One of the most powerful and natural means for human beings to communicate their emotions, feelings and intentions is a Facial Expression. Facial Expressions and other gestures, help convey non-verbal communication cues in face-to-face interactions. These cues can also complement speech by helping the listener to elicit the meaning of spoken words [1]. Automatic Facial Expression Recognition (FER) has attracted the attention of many researchers due to its potential applications in areas like Human Computer Interaction (HCI), Sign Language Recognition (SLR), Virtual Reality (VR) Systems, etc. Automatic FER could bring facial expressions into manmachine interaction as a new modality and make the integration tighter and more efficient [2]. Research on automatic FER addresses the problem of representing and categorizing the static or dynamic characteristics of deformations of facial components and their spatial relations, or changes in the pigmentation of the face [1].
Extraction of proper and sufficient features from the facial image is the most important step for effective FER. Facial feature extractors should be selected in such a way that they help to derive a set of features from original facial image which would minimize the intra-class differences and maximize the inter-class variations. Two main types of approaches have been used by researchers for extracting facial features in FER, one is based on face geometry while the other uses textural information of the facial image. In geometry based feature extraction techniques, face shape and location of facial components are used for defining feature vectors. Various 2D, 3D models and Facial Action Coding Systems (FACS) are used to describe the face structure [3] [4] [5]. The geometry based models require reliable and accurate feature detection / tracking [6]. They exhibit high recognition efficiency but are time and memory demanding. Appearance based techniques extract and use either holistic or local features for FER. Holistic features are extracted using various techniques such as Gabor filters [7] [8], Principle Component Analysis (PCA) [1] [4], Independent Component Analysis (ICA) [9], Linear Discriminant Analysis (LDA) [10], etc. Local feature extraction approaches predominantly use Local Binary Patterns (LBP) [11] or its variants [12] [13] [14] [15] to describe texture of the face. Higher order autocorrelation like features [16], Local PCA [1], Local LDA [1], etc. have also been used by researchers besides LBP. Amongst all the feature extraction techniques, LBP method has become quite popular due to its simplicity, impressive computational efficiency and good texture discriminative property [17].

As facial images can be differentiated from other textural images in the sense that they exhibit specific information as regards expressions around certain face regions (such as areas surrounding the eyes, nose, mouth, forehead and chin), efforts need to be done on augmenting the LBP with specific facial expression related information. This paper reports one such effort made to strengthen the LBP so that its suitability for application to facial expression recognition can be enhanced.

\section{REVIEW OF LBP}

The original LBP operator was introduced by Ojala et al. [18] for texture description. The LBP operator labels the pixels $f_{p}$ $(\mathrm{p}=0,1, \ldots .7)$ of an image by thresholding a $3 \times 3$ neighbourhood of each pixel with the value of the centre pixel $f_{c}$ and considering the result as a binary number $S\left(f_{p}-f_{c}\right)$ as shown in figure 1, where:

$\mathrm{S}\left(f_{\mathrm{p}}-f_{\mathrm{c}}\right)=\left\{\begin{array}{l}1 \text { if } f_{\mathrm{p}} \geq f_{\mathrm{c}} \\ 0 \text { otherwise }\end{array}\right.$ 
Then, by assigning a binomial factor $2^{p}$ for each $S\left(f_{p}-f_{c}\right)$, the LBP is computed as:

$$
\mathrm{LBP}=\sum_{p=0}^{7} S\left(f_{p}-f_{c}\right) 2^{p}
$$

LBPs have proven to be very effective for image representation and have been applied to visual inspection, motion detection and outdoor scene analysis. The LBPs are tolerant against monotonic illumination changes and detect many texture primitives like spot, line end, edge, corner etc. typically accumulated into a ( 256 bin) histogram over a region to capture local texture information [15].

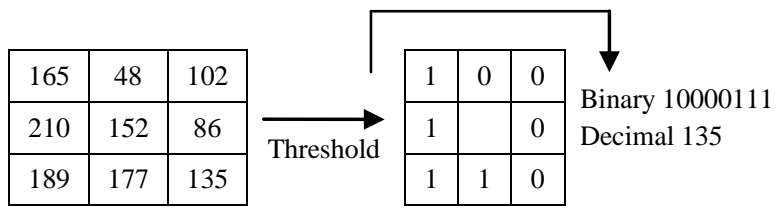

Figure 1: Basic LBP Operator (Adapted from [6])

To reduce the size of the histogram and hence the size of feature vector, Ojala et al. [19] introduced Uniform Patterns. Block LBP histograms were introduced by Ahonen et al. [20] which carry information about the local texture along with global shape. In basic LBP, no specific consideration is given for those regions of the face that would help discriminate inter-class expressions from each other. Shan et al. [6] used a weighting approach to highlight the probable feature existing regions like eyes and mouth. They, however, also clarify that the weight set adopted by them is decided empirically based on observations. Moore and Bowden [15] used LBP for multiview (frontal and profile) FER and investigated the performance of variations of LBPs like Rotational Invariant LBP, Magnitude LBP, Multi-scale LBP and Local Gabor Binary Patterns (LGBP).

Although, LBP has been reported to perform quite well for facial expression recognition, it is felt by the authors that LBP basically treats facial images as texture and therefore its performance with specific reference to FER can be enhanced by incorporating information (in some form) regarding facial components that would help differentiate inter-class expressions and group intra-class expressions.

\section{RADIAL LBP}

Use of LBPs particularly for facial expression extraction needs to take into consideration the pixel value variations exhibited in different directions or regions when different expressions are subjected to feature extraction. Two different approaches have been envisaged and proposed in this paper with the above objective in mind. The first approach considers drawing radial lines at fixed angle intervals from centre of an image to the boundary of the image with an assumption that most of these radial lines would encounter different patterns when subject to different expression images. For example, a radial line drawn at certain angle over a "neutral expression" image would encounter different pixel intensity variations as compared to a radial line drawn at the same angle over an "anger expression" image. Figure 2 (a) represents one image representing "neutral expression" from the JAFFE database superimposed with a line drawn at $260^{\circ}$ from the centre of the image to a point on the boundary of the image. Figure 2 (b) represents the same line superimposed on another image representing "anger expression" from the same database. If the LBP values are determined for each pixel on these lines, then it can clearly be observed that they would present substantially different representations for the two images.
Figures 3 (a) and (b) represent the above facial images with radial lines drawn at some specific incremental angle $(\beta)$. LBP (referred to as Radial LBP / RLBP hereafter) values for each such radial line drawn on a facial image are computed and represented as a histogram. All such histograms (one for each line) are concatenated to get the feature vector for that facial image. If an incremental angle $(\beta)$ of $10^{\circ}$ is considered then 36 radial lines shall be drawn and therefore each image would have a feature vector of size ' $36 \times \mathrm{N}$ ' where $\mathrm{N}$ represents the number of bins of the histograms. Using LBP values of such radial lines in deriving feature vector for feature extraction and subsequent classification is based on the consideration that pattern exhibited by RLBP values along those radial lines would sufficiently represent the regions containing useful information for expression classification.

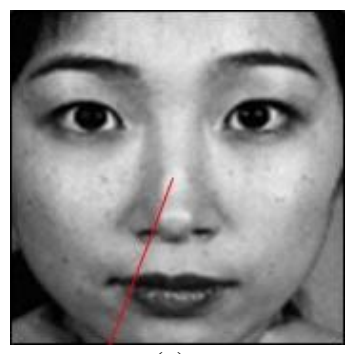

(a)

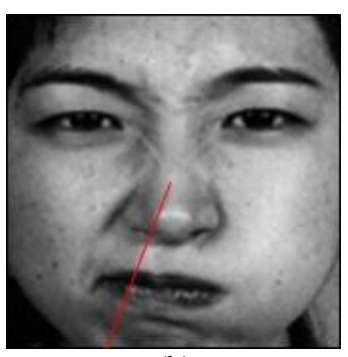

(b)
Figure 2: Radial Line drawn over (a) Neutral Expression Image (b) Anger Expression Image

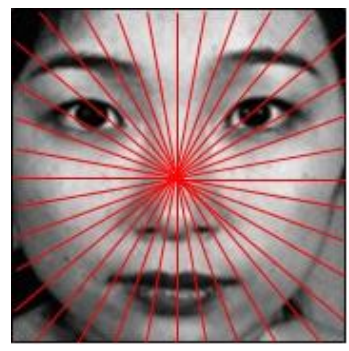

(a)

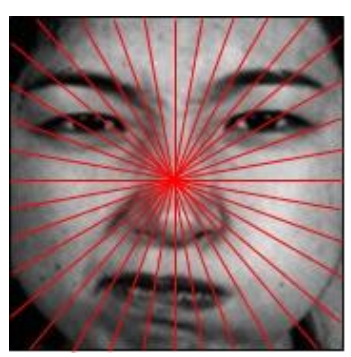

(b)
Figure 3: Radial lines drawn with an incremental angle ' $\beta$ ' over (a) Neutral Expression Image (b) Anger Expression Image

The second approach is based on the presumption that most of the facial expressions can be represented in terms of features of the areas surrounding eyes, nose and mouth. Each facial image is segmented, therefore, into four sub images by extracting left eye, right eye, nose and mouth. A simple region growing algorithm is used for the extraction of these four sub images from each of the facial images. Then, RLBP values are computed for each radial line of each sub image. Individual histogram is plotted for each radial for each of the four sub images of a facial image. Then all the histograms are concatenated to obtain the feature vector as shown in figure 4 .

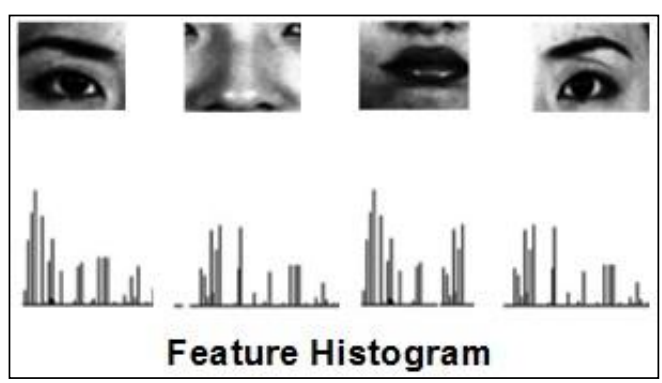

Figure 4: Feature Histogram obtained by concatenating sub image histograms 
Use of RLBP for each sub image is again based on the consideration that pattern exhibited by RLBP values along those radial lines would sufficiently represent the regions containing useful information for expression classification. Therefore, each image would now have a feature vector of size ' $36 \times 4 * \mathrm{~N}$ ' if radial lines are drawn at $10^{\circ}$ incremental angle where 4 represents number of sub images and $\mathrm{N}$ represents number of bins.

\section{EXPERIMENTATION}

It is proposed in this paper to evaluate the performance of feature vectors based on Radial LBPs. As two approaches have been proposed, two sets of experiments (i.e. one for RLBP with full facial image (WI) and the other for RLBP with four sub images (ENM)) have been conducted. Five different incremental angle values were considered to understand how recognition efficiency would be affected by the angle at which radial lines are drawn. Two low values (namely $2^{\circ}$ and $5^{\circ}$ ) and three high values (namely $10^{\circ}, 12^{\circ}$ and $15^{\circ}$ ) of $\beta$ were taken for the purpose of experimentation. Besides, we intend to have as low a value of number of bins $(\mathrm{N})$ as possible to reduce size of the feature vector. However, before fixing the value of $\mathrm{N}$, we varied its value also during the experiments to identify which value caters to better recognition efficiency. Four values namely $8,12,16$ and 20 were considered for $\mathrm{N}$. The database used for these experiments consists of 210 facial images taken from JAFFE database with 10 persons, 7 expressions namely 'Anger', 'Disgust', 'Fear', 'Happy', 'Neutral', 'Sad' and 'Surprise' and 3 replications of each expression.

SVM has been used in the work reported in this paper for the purpose of classification. SVM does binary decisions. Multi class classification here is performed using binary classifiers along with a voting scheme. A test image, whose expression is not known, is subjected to a binary classification using SVM say between a pair of 'Anger' and 'Disgust'. The outcome of this classification whether 'Anger' or 'Disgust' is then subjected to pairwise comparison with remaining 5 expressions one after the other. Each time a pairwise comparison is done, one of the expressions in the pair is the one classified by SVM in the previous comparison. The final outcome after all pairwise comparisons is declared as the recognized expression.

Major steps of the experimentation are enlisted below:

1. For each pair of $\beta$ and $N$ values from the sets:

$S_{\beta}=\{2,5,10,12,15\} ; S_{N}=\{8,12,16,20\}$, perform the

following steps.

2. Extract features of all the test and reference images. For experimentation set 1 , feature vector would be of size ' $(360 / \beta) \times N$ ' whereas for experimentation set 2 , it would be of size ' $(360 / \beta) \times 4 * N$ '.

3. Segregate feature vectors of the 210 images so that 3 sets of 70 images each get grouped in such a way that each set consists of one replication of the 7 expressions of all 10 persons. Define one set (not selected earlier) as the set of test images and define the remaining two sets together as reference images.

4. Use SVM for classifying each of the test feature vector. As SVM does pairwise comparison, a test feature vector (whose expression group is not known) is subject to a series of pairwise comparisons. At each such comparison, the test image would be checked against a set of 40 reference feature vectors out of the available 140. The 40 feature vectors are the ones which correspond to the pair of expressions under comparison.

Repeat steps 3 through 4 till each of the three sets of 70 images are subjected to classification as the test samples.

\section{RESULTS}

The results are presented for experiment set 1 and 2 separately. The results include representation of overall recognition efficiency achieved (average results over three cycles are considered as the recognition rate) and confusion matrix depicting the recognition efficiency of individual expressions along with the misrecognition occurred.

\subsection{Results of Experiment Set 1}

For different combination of incremental angle $(\beta)$ and number of bins $(\mathrm{N})$, the feature vector size can be represented as $(360 / \beta) \times \mathrm{N}($ table 1$)$.

Table 1: Feature Vector Size for different combinations of $\beta$ and $N$ over full facial image (WI)

\begin{tabular}{|c|c|c|c|c|c|}
\hline & $\boldsymbol{\beta = 2}$ & $\boldsymbol{\beta = 5}$ & $\boldsymbol{\beta = 1 0}$ & $\boldsymbol{\beta}=\mathbf{1 2}$ & $\boldsymbol{\beta}=\mathbf{1 5}$ \\
\hline $\mathbf{N = \mathbf { 8 }}$ & 1440 & 576 & 288 & 240 & 192 \\
\hline $\mathbf{N}=\mathbf{1 2}$ & 2160 & 864 & 432 & 360 & 288 \\
\hline $\mathbf{N}=\mathbf{1 6}$ & 2880 & 1152 & 576 & 480 & 384 \\
\hline $\mathbf{N}=\mathbf{2 0}$ & 3600 & 1440 & 720 & 600 & 480 \\
\hline
\end{tabular}

Overall recognition efficiency obtained in percentage is presented in figure 5 and table 2 .

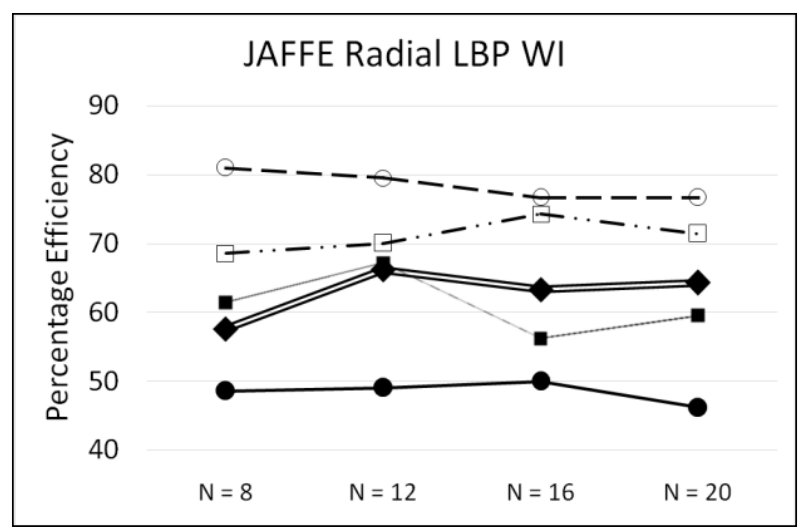

Figure 5: Overall Recognition Efficiency obtained by using RLBP over full facial images

Table 2: Overall Recognition Efficiency values (\%)

\begin{tabular}{|c|c|c|c|c|c|}
\hline & $\boldsymbol{\beta = 2}$ & $\boldsymbol{\beta}=\mathbf{5}$ & $\boldsymbol{\beta}=\mathbf{1 0}$ & $\boldsymbol{\beta}=\mathbf{1 2}$ & $\boldsymbol{\beta}=\mathbf{1 5}$ \\
\hline $\mathbf{N = \mathbf { 8 }}$ & 80.95 & 68.57 & 57.62 & 61.43 & 48.57 \\
\hline $\mathbf{N}=\mathbf{1 2}$ & 79.52 & 70.00 & 66.19 & 67.14 & 49.05 \\
\hline $\mathbf{N}=\mathbf{1 6}$ & 76.67 & 74.29 & 63.33 & 56.19 & 50.00 \\
\hline $\mathbf{N}=\mathbf{2 0}$ & 76.67 & 71.43 & 64.29 & 59.52 & 46.19 \\
\hline
\end{tabular}

It can be observed from figure 5 and table 2 , that recognition efficiency has been obtained for $\beta=2$ and that higher values of $\beta\left(10^{\circ}, 12^{\circ}\right.$ and $\left.15^{\circ}\right)$ exhibited significantly poor performance. The results are, however, encouraging as regards the applicability of RLBP to facial feature extraction because RLBP has exhibited quite good performance in spite of the low feature vector size. Application of simple LBP over 
whole image has resulted in an efficiency of only $40 \%$ while the minimum efficiency here is 46.19 . So potential of RLBP can be further explored.

For an in depth analysis of the performance of RLBP, we present here (table 3) the confusion matrices for the four different combinations of $\beta$ and $\mathrm{N}$. The highlighted entries in the confusion matrix for all the combinations of $\beta$ and $\mathrm{N}$ show recognition efficiency of corresponding expressions that are far below the overall average efficiency of the corresponding combination of $\beta$ and N. It can be seen that RLBP exhibits a little less efficiency in recognizing the 'Disgust', 'Fear', 'Sad' and 'Surprise'. In particular, expression 'Sad' was consistently recognized below overall average.

Table 3: Confusion Matrices for four combinations of $\beta$ and $\mathrm{N}$ obtained for the whole facial image

\begin{tabular}{|l|c|c|c|c|c|c|c|}
\hline $\begin{array}{c}\boldsymbol{\beta}=\mathbf{2}, \\
\mathbf{N}=8\end{array}$ & Anger & Disgust & Fear & Happy & $\begin{array}{c}\text { Neut- } \\
\text { ral }\end{array}$ & Sad & $\begin{array}{c}\text { Surp- } \\
\text { rise }\end{array}$ \\
\hline Anger & 93.33 & 3.33 & 0.00 & 0.00 & 0.00 & 3.33 & 0.00 \\
\hline Disgust & 6.67 & 80.00 & 10.00 & 0.00 & 0.00 & 3.33 & 0.00 \\
\hline Fear & 3.33 & 6.67 & 76.67 & 0.00 & 0.00 & 6.67 & 6.67 \\
\hline Happy & 0.00 & 0.00 & 0.00 & 90.00 & 6.67 & 3.33 & 0.00 \\
\hline Neutral & 0.00 & 0.00 & 3.33 & 0.00 & 90.00 & 6.67 & 0.00 \\
\hline Sad & 10.00 & 0.00 & 16.67 & 3.33 & 6.67 & 60.00 & 3.33 \\
\hline Surprise & 0.00 & 3.33 & 6.67 & 3.33 & 6.67 & 3.33 & 76.67 \\
\hline
\end{tabular}

\begin{tabular}{|l|c|c|c|c|c|c|c|}
\hline $\begin{array}{c}\boldsymbol{\beta}=\mathbf{5}, \\
\mathbf{N = 1 6}\end{array}$ & Anger & Disgust & Fear & Happy & $\begin{array}{c}\text { Neut- } \\
\text { ral }\end{array}$ & Sad & $\begin{array}{c}\text { Surp- } \\
\text { rise }\end{array}$ \\
\hline Anger & 80.00 & 13.33 & 0.00 & 0.00 & 3.33 & 3.33 & 0.00 \\
\hline Disgust & 13.33 & 66.67 & 13.33 & 0.00 & 0.00 & 3.33 & 3.33 \\
\hline Fear & 6.67 & 13.33 & 56.67 & 0.00 & 3.33 & 13.33 & 6.67 \\
\hline Happy & 0.00 & 0.00 & 0.00 & 83.33 & 10.00 & 6.67 & 0.00 \\
\hline Neutral & 3.33 & 6.67 & 0.00 & 3.33 & 83.33 & 3.33 & 0.00 \\
\hline Sad & 3.33 & 0.00 & 13.33 & 6.67 & 3.33 & 70.00 & 3.33 \\
\hline Surprise & 0.00 & 3.33 & 3.33 & 6.67 & 3.33 & 3.33 & 80.00 \\
\hline
\end{tabular}

\begin{tabular}{|l|c|c|c|c|c|c|c|}
\hline $\begin{array}{c}\boldsymbol{\beta}=10, \\
\mathbf{N}=12\end{array}$ & Anger & Disgust & Fear & Happy & $\begin{array}{c}\text { Neut- } \\
\text { ral }\end{array}$ & Sad & $\begin{array}{c}\text { Surp- } \\
\text { rise }\end{array}$ \\
\hline Anger & 66.67 & 13.33 & 6.67 & 3.33 & 6.67 & 3.33 & 0.00 \\
\hline Disgust & 13.33 & 53.33 & 16.67 & 0.00 & 10.00 & 6.67 & 0.00 \\
\hline Fear & 6.67 & 3.33 & 66.67 & 0.00 & 3.33 & 10.00 & 10.00 \\
\hline Happy & 0.00 & 0.00 & 3.33 & 80.00 & 6.67 & 6.67 & 3.33 \\
\hline Neutral & 3.33 & 0.00 & 6.67 & 13.33 & 66.67 & 6.67 & 3.33 \\
\hline Sad & 0.00 & 6.67 & 13.33 & 3.33 & 16.67 & 53.33 & 6.67 \\
\hline Surprise & 0.00 & 0.00 & 6.67 & 6.67 & 3.33 & 6.67 & 76.67 \\
\hline
\end{tabular}

\begin{tabular}{|l|c|c|c|c|c|c|c|}
\hline $\begin{array}{c}\boldsymbol{\beta}=12, \\
\mathbf{N}=20\end{array}$ & Anger & Disgust & Fear & Happy & $\begin{array}{c}\text { Neut- } \\
\text { ral }\end{array}$ & Sad & $\begin{array}{c}\text { Surp- } \\
\text { rise }\end{array}$ \\
\hline Anger & 73.33 & 6.67 & 6.67 & 3.33 & 10.00 & 0.00 & 0.00 \\
\hline Disgust & 13.33 & 60.00 & 6.67 & 3.33 & 0.00 & 13.33 & 3.33 \\
\hline Fear & 3.33 & 13.33 & 53.33 & 3.33 & 0.00 & 20.00 & 6.67 \\
\hline Happy & 3.33 & 3.33 & 3.33 & 66.67 & 16.67 & 3.33 & 3.33 \\
\hline Neutral & 10.00 & 3.33 & 0.00 & 13.33 & 63.33 & 3.33 & 6.67 \\
\hline Sad & 3.33 & 6.67 & 16.67 & 3.33 & 23.33 & 46.67 & 0.00 \\
\hline Surprise & 3.33 & 3.33 & 16.67 & 3.33 & 16.67 & 3.33 & 53.33 \\
\hline
\end{tabular}

It can be concluded from the results of this experimentation that although RLBP was able to capture some of the expression specific information, it failed to adequately differentiate all the expressions.

\subsection{Results of Experiment Set 2}

Experiment set 2, as mentioned earlier, segments four sub images (namely the two eyes, nose and mouth) from the full facial image with an intention of augmenting the LBPs with regional information specific to the facial expression. For different combination of incremental angle $(\beta)$ and number of bins $(\mathrm{N})$, the feature vector size can be represented as $(360 / \beta) \times 4 * \mathrm{~N}$ (table 4$)$.

Table 4: Feature Vector Size for different combinations of $\beta$ and $N$ considering four sub images (ENM)

\begin{tabular}{|c|c|c|c|c|c|}
\hline & $\boldsymbol{\beta = 2}$ & $\boldsymbol{\beta = 5}$ & $\boldsymbol{\beta}=\mathbf{1 0}$ & $\boldsymbol{\beta}=\mathbf{1 2}$ & $\boldsymbol{\beta}=\mathbf{1 5}$ \\
\hline $\mathbf{N = \mathbf { 8 }}$ & 5760 & 2304 & 1152 & 960 & 768 \\
\hline $\mathbf{N}=\mathbf{1 2}$ & 8640 & 3456 & 1728 & 1440 & 1152 \\
\hline $\mathbf{N}=\mathbf{1 6}$ & 11520 & 4608 & 2304 & 1920 & 1536 \\
\hline $\mathbf{N}=\mathbf{2 0}$ & 14400 & 5760 & 2880 & 2400 & 1920 \\
\hline
\end{tabular}

Overall recognition efficiency obtained in percentage is presented in figure 6 and table 5. It can again be observed from figure 6 and table 5 that in general $\mathrm{N}$ value of 12 gave better recognition efficiency. Although, higher values of $\beta$ $\left(10^{\circ}, 12^{\circ}\right.$ and $\left.15^{\circ}\right)$ still exhibited less performance, the results are significantly better than the results obtained when RLBP was applied over full facial image. Minimum overall recognition efficiency obtained now has shot up to $63.33 \%$. This clearly indicates that augmenting the LBPs with expression specific information from the areas near the two eyes, nose and mouth has a significant impact on the performance of LBPs. It can be said in other words that augmentation of geometry based information into appearance based features has resulted in better efficiency.

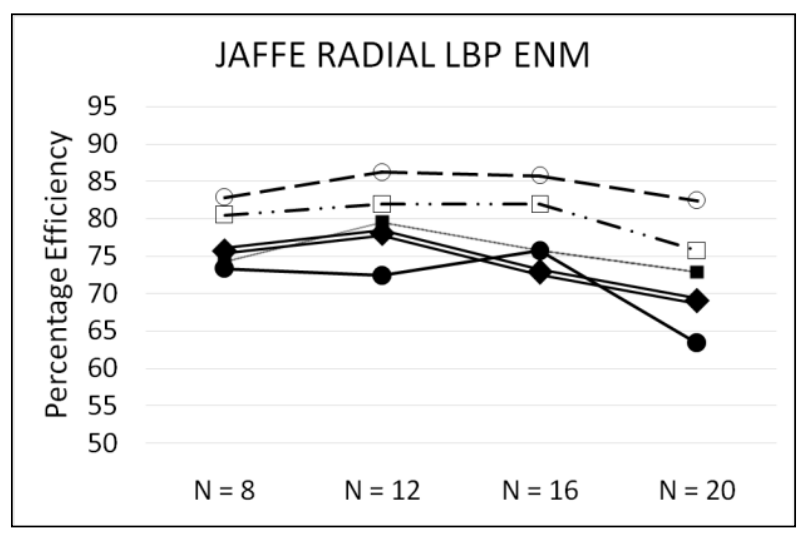

Figure 6: Overall Recognition Efficiency obtained by using RLBP over four sub images

Table 5: Overall Recognition Efficiency values (\%)

\begin{tabular}{|c|c|c|c|c|c|}
\hline & $\boldsymbol{\beta}=\mathbf{2}$ & $\boldsymbol{\beta}=\mathbf{5}$ & $\boldsymbol{\beta}=\mathbf{1 0}$ & $\boldsymbol{\beta}=\mathbf{1 2}$ & $\boldsymbol{\beta}=\mathbf{1 5}$ \\
\hline $\mathbf{N}=\mathbf{8}$ & 82.86 & 80.48 & 75.71 & 74.29 & 73.33 \\
\hline $\mathbf{N}=\mathbf{1 2}$ & 86.19 & 81.90 & 78.10 & 79.52 & 72.38 \\
\hline $\mathbf{N}=\mathbf{1 6}$ & 85.71 & 81.90 & 72.86 & 75.71 & 75.71 \\
\hline $\mathbf{N}=\mathbf{2 0}$ & 82.38 & 75.71 & 69.05 & 72.86 & 63.33 \\
\hline
\end{tabular}

For a deeper look into the performance of RLBP applied over sub images, we present the confusion matrices for four different combinations of $\beta$ and $\mathrm{N}$ in table 6 . The highlighted entries in the confusion matrix for all the combinations of $\beta$ and $\mathrm{N}$ again represent recognition efficiency far below the overall average efficiency of the corresponding combination of $\beta$ and N. Here only two expressions namely 'Fear' and 'Sad' could not be recognized properly. Besides, the overall efficiency of recognition has also gone up. 


\section{CONCLUSIONS}

Based on the above two experimentations, we can conclude the following:

RLBP directly applied over the full facial image gives higher recognition efficiency for lower values of $\beta\left(2^{\circ}\right.$ and $\left.5^{\circ}\right)$. Besides, recognition of certain expressions such as 'Fear' and 'Sad' was particularly poor. The feature vector size for RLBP applied over full facial image was however comparatively quite small and thereby resulting in lesser time / memory requirement.

Table 6: Confusion Matrices for four combinations of $\beta$ and $\mathbf{N}$ obtained for the four sub images

\begin{tabular}{|l|c|c|c|c|c|c|c|}
\hline $\begin{array}{c}\boldsymbol{\beta}=\mathbf{2}, \\
\text { N=8 }\end{array}$ & Anger & Disgust & Fear & Happy & $\begin{array}{c}\text { Neut- } \\
\text { ral }\end{array}$ & Sad & $\begin{array}{c}\text { Surp- } \\
\text { rise }\end{array}$ \\
\hline Anger & 93.33 & 3.33 & 0.00 & 0.00 & 0.00 & 3.33 & 0.00 \\
\hline Disgust & 10.00 & 83.33 & 3.33 & 0.00 & 0.00 & 3.33 & 0.00 \\
\hline Fear & 0.00 & 6.67 & 76.67 & 0.00 & 6.67 & 3.33 & 6.67 \\
\hline Happy & 0.00 & 3.33 & 0.00 & 80.00 & 13.33 & 3.33 & 0.00 \\
\hline Neutral & 0.00 & 0.00 & 3.33 & 0.00 & 90.00 & 3.33 & 3.33 \\
\hline Sad & 3.33 & 0.00 & 16.67 & 6.67 & 3.33 & 70.00 & 0.00 \\
\hline Surprise & 0.00 & 0.00 & 0.00 & 3.33 & 10.00 & 0.00 & 86.67 \\
\hline
\end{tabular}

\begin{tabular}{|l|c|c|c|c|c|c|c|}
\hline $\begin{array}{c}\boldsymbol{\beta}=\mathbf{5}, \\
\mathbf{N = 1 6}\end{array}$ & Anger & Disgust & Fear & Happy & $\begin{array}{c}\text { Neut- } \\
\text { ral }\end{array}$ & Sad & $\begin{array}{c}\text { Surp- } \\
\text { rise }\end{array}$ \\
\hline Anger & 90.00 & 3.33 & 0.00 & 0.00 & 6.67 & 0.00 & 0.00 \\
\hline Disgust & 13.33 & 80.00 & 3.33 & 0.00 & 0.00 & 3.33 & 0.00 \\
\hline Fear & 0.00 & 6.67 & 73.33 & 0.00 & 6.67 & 6.67 & 6.67 \\
\hline Happy & 0.00 & 0.00 & 0.00 & 80.00 & 16.67 & 3.33 & 0.00 \\
\hline Neutral & 3.33 & 0.00 & 0.00 & 6.67 & 86.67 & 0.00 & 3.33 \\
\hline Sad & 0.00 & 0.00 & 13.33 & 6.67 & 6.67 & 73.33 & 0.00 \\
\hline Surprise & 0.00 & 0.00 & 0.00 & 3.33 & 6.67 & 0.00 & 90.00 \\
\hline
\end{tabular}

\begin{tabular}{|l|c|c|c|c|c|c|c|}
\hline $\begin{array}{c}\boldsymbol{\beta}=10, \\
\mathbf{N = 1 2}\end{array}$ & Anger & Disgust & Fear & Happy & $\begin{array}{c}\text { Neut- } \\
\text { ral }\end{array}$ & Sad & $\begin{array}{c}\text { Surp- } \\
\text { rise }\end{array}$ \\
\hline Anger & 90.00 & 6.67 & 0.00 & 0.00 & 0.00 & 3.33 & 0.00 \\
\hline Disgust & 13.33 & 76.67 & 3.33 & 0.00 & 0.00 & 6.67 & 0.00 \\
\hline Fear & 0.00 & 10.00 & 56.67 & 3.33 & 10.00 & 13.33 & 6.67 \\
\hline Happy & 0.00 & 0.00 & 0.00 & 76.67 & 20.00 & 3.33 & 0.00 \\
\hline Neutral & 3.33 & 0.00 & 0.00 & 6.67 & 90.00 & 0.00 & 0.00 \\
\hline Sad & 3.33 & 0.00 & 13.33 & 3.33 & 10.00 & 70.00 & 0.00 \\
\hline Surprise & 0.00 & 0.00 & 0.00 & 3.33 & 10.00 & 0.00 & 86.67 \\
\hline
\end{tabular}

\begin{tabular}{|l|c|c|c|c|c|c|c|}
\hline $\begin{array}{c}\boldsymbol{\beta}=12, \\
\text { N=20 }\end{array}$ & Anger & Disgust & Fear & Happy & $\begin{array}{c}\text { Neut- } \\
\text { ral }\end{array}$ & Sad & $\begin{array}{c}\text { Surp- } \\
\text { rise }\end{array}$ \\
\hline Anger & 83.33 & 3.33 & 3.33 & 0.00 & 10.00 & 0.00 & 0.00 \\
\hline Disgust & 10.00 & 76.67 & 6.67 & 0.00 & 0.00 & 6.67 & 0.00 \\
\hline Fear & 3.33 & 10.00 & 63.33 & 3.33 & 6.67 & 6.67 & 6.67 \\
\hline Happy & 0.00 & 0.00 & 0.00 & 73.33 & 13.33 & 10.00 & 3.33 \\
\hline Neutral & 3.33 & 0.00 & 10.00 & 6.67 & 70.00 & 6.67 & 3.33 \\
\hline Sad & 0.00 & 3.33 & 20.00 & 6.67 & 10.00 & 60.00 & 0.00 \\
\hline Surprise & 0.00 & 0.00 & 0.00 & 3.33 & 13.33 & 0.00 & 83.33 \\
\hline
\end{tabular}

Results obtained on applying RLBP over four sub images of the full facial image are very encouraging. Thus, augmenting LBPs with region based expression specific information can be seen as hybrid geometry and appearance based approach. Obviously, benefits of both approaches can be combined for better performance.

RLBP basically focuses on areas where features are expected to be present and does not consider the complete facial region which is otherwise considered by LBP technique.
Radial LBPs do help in augmenting the basic LBPs with facial expression specific information.

Efforts can further be put in the direction to modify or augment the RLBP features so as to have increase in the recognition efficiency.

Radial LBP does definitely capture the differences in expression particularly when consider over the four sub regions. However, the feature vector size becomes large when the sub regions are considered. Looking to the potential of RLBP, it is felt that further improvisation in the efforts to capture the variations in images with reference to facial expression recognition are warranted. Future scope to this work may consider augmenting the RLBP through additional features that have potential of capturing the expressions. However, to support real time recognition care should be taken to keep the feature vector size as small as possible.

\section{REFERENCES}

[1] Chibelushi C. C., Bourel F., 2002. Facial Expression Recognition: A Brief Tutorial Overview, IEEE Conference, pp 1-5

[2] Patic M., Rothkrantz L. J. M., 2000. Automatic Analysis of Facial Expressions: The State of the Art, IEEE Transactions on Pattern and Machine Intelligence, Vol. 22, No. 12, pp 1424-1443

[3] Fasel B., Luettin J., 2003. Automatic Facial Expression Analysis: A Survey, Pattern Recognition, A journal by Pattern Recognition Society, Published by Elsevier Science, Vol. 36, pp 259-275.

[4] Kotsia I. and Pitas I., 2007. Facial Expression Recognition in Image Sequences Using Geometric Deformation Features and Support Vector Machines, IEEE Transactions on Image Processing, Vol. 16, No.1, pp 172-187.

[5] Chibelushi C. C. and Bourel F., 2004. Hierarchical Multistream Recognition of Facial Expressions, IEEE Proceedings-Vis. Image Signal Process, Vol. 151, No.4, pp307-313.

[6] Shan C., Gong S. and McOwan P.W., 2009. Facial expression recognition based on Local Binary Patterns: A comprehensive study, Elsevier, Image and Vision Computing, Vol. 27, pp 803-816.

[7] Lajevardi S. M. and Lech M., 2008. Averaged Gabor Filter Features for Facial Expression Recognition, IEEE Computer Society, Digital Image Computing: Techniques and Applications, pp71-76.

[8] Seyedehsamaneh S., Yun Y. W. and Khwang T. E., 2011. Person Independent Facial Expression Analysis using Gabor Features and Genetic Algorithm, IEEE Int. Conf. on ICS.

[9] ZiaUddin Md., Lee J. J. and Kim T. S., 2009. An Enhanced Independent Component Based Human Facial Expression Recognition from Video, IEEE Transactions on Consumer Electronics, Vol. 55, No. 4, pp 2216-2224.

[10] Yi J., Mao X., Chen L., Xue Y., Compare A.,2013. Facial Expression Recognition Considering Individual Differences in Facial Structure and Texture, IET Computer Vision, pp 1-12. 
[11] Shan C., Gong S. and McOwan P.W., 2005. Robust Facial Expression Recognition Using Local Binary Patterns, IEEE.

[12] Zhao Q., Pan B., Pan J., Tang Y ., 2008. Facial Expression Recognition Based on Fusion of Gabor and LBP Features, IEEE Int. Conf. on Wavelet Analysis and Pattern Recognition, Hong Kong, pp 362-367.

[13] He L., Zou C., Zhao L. and Hu D., 2005. An Enhanced LBP Feature Based on Facial Expression Recognition, Proceedings of 2005 IEEE Engg. in Medicine and Biology 27th Annual Conf. Shanghai, China, pp 33003303.

[14] Lajevardi S. M. and Hussain Z. M., 2009. Facial Expression Recognition using Log-Gabor Filters and Local Binary Pattern Operators, Int. Conference on Communication, Computer and Power, Muscat, pp 349353.

[15] Moore S. and Bowden R., 2011. Local Binary Patterns for Multi-view Facial Expression Recognition, Elsevier, Computer Vision and Image Understanding, 115, pp 541-558.
[16] Lajevardi S. M. and Hussain Z. M., 2009. Novel Higher Order Local Autocorrelation-Like Feature Extraction Methodology for Facial Expression Recognition, IET Image Processing, pp 114-119.

[17] Liu L., Zhao L. Long Y. Kuang G. and Fieguth P., 2012. Extended Local Binary Patterns for Texture Classification, Image and Vision Computing, Elsevier, Vol. 30, pp 86-89.

[18] Ojala T., Pietikäinen M., Harwood D., 1996. A comparative study of texture measures with classification based on featured distribution, Pattern Recognition, 29 (1), pp 51-59.

[19] Ojala T., Pietikäinen M., Mäenpää T., 2002 Multiresolution gray-scale and rotation invariant texture classification with local binary patterns, IEEE Transactions on Pattern Analysis and Machine Intelligence, 24 (7), 971-987.

[20] Ahonen T., Halid A., Pietikäinen M., 2004. Face Recognition using Local Binary Patterns, European Conference on Computer Vision (ECCV). 